\title{
Numerical solution of nonlinear system of integro-differential equations using Chebyshev wavelets
}

\author{
H. AMINIKHAH AND S. HOSSEINI
}

\begin{abstract}
This paper introduces an approach for obtaining the numerical solution of the linear and nonlinear integro-differential equations using Chebyshev wavelets approximations. Illustrative examples have been discussed to demonstrate the validity and applicability of the technique and the results have been compared with the exact solution. Comparison of the approximate solution with exact solution shows that the used method is effectiveness and practical for classes of linear and nonlinear system of integrodifferential equations.
\end{abstract}

Mathematics Subject Classification 2010. Primary 65T60; Secondary 05E35, 45J05.

Additional Key Words and Phrases: system of integro-differential equations; Chebyshev wavelets; operational matrix integration

\section{INTRODUCTION}

Many mathematical formulations of physical phenomena contain integrodifferential equations. These equations arise in many fields like fluid dynamics, theoretical physics, epidemic models, biological models, chemical kinetics, nanohydrodynamics, glass-forming process, drop wise condensation, wind ripple in the desert and many other disciplines lead to nonlinear integro-differential equations [17]. For solving these equations several numerical approaches have been proposed, an overview can be found in the monograph [8]. Wavelets theory is relatively new in mathematical researches. In recent years, wavelets have found their way into different field of science and engineering [9-13]. Wavelets permit the accurate representation of a variety of functions and operators. The common wavelet which has been used is Chebyshev wavelets. The main characteristic of this technique is that it reduces these problems to those of solving a system of algebraic equations. An extension of Chebyshev wavelets method for solving nonlinear systems of 
intego-differential equations is the novelty of this paper. The general form of system of integro-differential equations can be presented as follows

$$
\begin{aligned}
& \sum_{k=1}^{m} F_{i k}\left(x, f_{1}(x), f_{1}^{\prime}(x), \ldots, f_{1}^{\left(m_{1}\right)}(x), \ldots, f_{n}(x), f_{n}^{\prime}(x), \ldots, f_{n}^{\left(m_{n}\right)}(x)\right) \\
& +\sum_{j=1}^{m^{\prime}} \int_{0}^{x} k_{i j}(x, t) G_{i j}\left(f_{1}(t), \ldots, f_{1}^{\left(m_{1}\right)}(t), \ldots, f_{n}(t), \ldots, f_{n}^{\left(m_{n}\right)}(t)\right) d t=g_{i}(x) \\
& \quad i=1,2, \ldots, n
\end{aligned}
$$

with initial conditions

$$
f_{i}^{(s)}(0)=\alpha_{i, s}, i=1,2, \ldots, n, s=0,1, \ldots, m_{i}-1
$$

where $f_{1}, f_{2}, \ldots, f_{n}$ are unknown functions that should be determined by the Chebyshev wavelets method.

The rest of the paper is organized as follows:

In Section 2, after describing the basic formulation of wavelets and the first kind Chebyshev wavelet, we derive the first kind Chebyshev wavelet operational matrix of the integration. In Section 3, the way to construct the Chebyshev wavelet technique for system of integro-differential equations are described. In Section 4 the proposed method is applied to some types of these equations. Conclusions are given in Section 5.

\section{THE FIRST KIND CHEBYSHEV WAVELET AND OPERATIONAL MATRIX OF THE INTEGRATION}

In this section, we use the first kind Chebyshev polynomial to construct the first kind Chebyshev wavelet and give some properties of this wavelet. 


\subsection{The first kind Chebyshev wavelet}

Wavelets constitute a family of functions constructed from dilation and translation of a single function called the mother wavelet [13-16]. When the dilation parameter $a$ and the translation parameter $b$ vary continuously we have following family of continuous wavelets as

$$
\psi_{a, b}(x)=|a|^{-\frac{1}{2}} \psi \frac{x-b}{a}, \quad a, b \in \mathbb{R}, a \neq 0 .
$$

If we take dilation and translation parameters $a^{-k}$ and $n b a^{-k}$, respectively where $a>1, b>0, n$, and $k$ are positive integers then we have the following family of discrete wavelets

$$
\psi_{k, n}(x)=|a|^{\frac{k}{2}} \psi\left(a^{k} x-n b\right)
$$

These functions are a wavelet basis for $L^{2}(\mathbb{R})$ and in special case $a=2$ and $b=1$ the functions $\psi_{k, n}(x)$ are an orthonormal basis.

Chebyshev wavelets $\quad \psi_{n, m}(x)=\psi(k, n, m, x) \quad$ have $\quad$ four $\quad$ arguments $n=1,2, \ldots, 2^{k-1}$ and $k$ is an arbitrary positive integer and $m$ is the order of Chebyshev polynomials of the first kind. They are defined on the interval $[0,1]$ as follows:

$$
\psi_{\mathrm{n}, \mathrm{m}}(x)=\psi(k, n, m, x)=\left\{\begin{array}{lc}
2^{\frac{k}{2}} \tilde{T}_{m}\left(2^{k} x-2 n+1\right), & \frac{n-1}{2^{k-1}} \leq x<\frac{n}{2^{k-1}} \\
0, & \text { otherwise }
\end{array}\right.
$$

where 
$\tilde{T}_{m}(x)= \begin{cases}\frac{1}{\sqrt{\pi}}, & m=0, \\ \sqrt{\frac{2}{\pi}} T_{m}(x), & m>0 .\end{cases}$

and $m=0,1, \ldots, M-1$ and $n=1,2, \ldots, 2^{k-1} . \quad T_{m}(x)$ are the famous Chebyshev polynomials of the first kind of degree $m$,which are orthogonal with respect to the weight function $W(x)=\frac{1}{\sqrt{1-x^{2}}}$ on the interval $[-1,1]$, and satisfy the following recursive formula:

$$
\left\{\begin{array}{l}
T_{0}(x)=1, T_{1}(x)=x, \\
T_{m+1}(x)=2 x T_{m}(x)-T_{m-1}(x), \quad m=1,2, \ldots
\end{array}\right.
$$

The set of Chebyshev wavelets is an orthogonal set with respect to the weight function $W_{n}(x)=W\left(2^{k} x-2 n+1\right)$.

\subsection{Function approximation}

A function $f(x)$ defined on the interval $[0,1]$ may be presented as

$$
f(x)=\sum_{n=1}^{\infty} \sum_{m=0}^{\infty} c_{n m} \psi_{n m}(x)
$$

The series representation of $f(x)$ in (6) is called a wavelet series and the wavelet coefficients $c_{n m}$ are given by $c_{n m}=\left(f(x), \psi_{n m}(x)\right)_{W_{n}(x)}$.

The convergence of the series 6 in $L^{2} \quad 0,1$ means that

$$
\lim _{s 1, s 2 \rightarrow \infty}\left\|f(x)-\sum_{n=1}^{s 1} \sum_{m=0}^{s 2} c_{n m} \psi_{n m}(x)\right\|=0
$$


Therefore one can consider the following truncated series for series (8)

$$
f(x) \simeq \sum_{n=1}^{2^{k-1}} \sum_{m=0}^{M-1} c_{n m} \psi_{n m}(x)=C^{T} \psi(x)
$$

where $\mathrm{C}$ and $\psi(x)$ are $2^{k-1} M \times 1$ matrices given by

$$
\begin{aligned}
C & =c_{10}, c_{11}, \ldots, c_{1, M-1}, c_{20}, c_{21}, \ldots, c_{2 M-1}, \ldots, c_{2^{k-1} 0}, \ldots, c_{2^{k-1} M-1}^{T} \\
& =c_{1}, c_{2}, \ldots, c_{M}, c_{M+1}, \ldots, c_{2^{k-1} M}^{T}
\end{aligned}
$$

and

$$
\begin{aligned}
\psi(x) & =\psi_{10}(x), \psi_{11}(x), \ldots, \psi_{1 M-1}(x), \psi_{20}(x), \psi_{21}(x), \ldots, \psi_{2 M-1}(x), \ldots, \psi_{2^{k-1} 0}(x), \ldots \psi_{2^{k-1} M-1}(x)^{T} \\
& =\psi_{1}(x), \psi_{2}(x), \ldots, \psi_{M}(x), \psi_{M+1}(x), \ldots, \psi_{2^{k-1} M}(x)^{T}
\end{aligned}
$$

The integration of the product of two Chebyshev wavelets vector functions with respect to the weight function $W_{n}(x)$, is derived as

$$
\int_{0}^{1} W_{n}(x) \psi(x) \psi^{T}(x) d x=I
$$

where $I$ is an identity matrix.

A function $f(x, y)$ defined on $0,1 \times 0,1$ can be approximated as the following

$$
f(x, y) \simeq \psi^{T}(x) K \psi(y)
$$

Here the entries of matrix $K=k_{i j} 2^{k-1} M \times 2^{k-1} M$ will be obtain by

$$
k_{i j}=\psi_{i}(x),\left(f(x, y), \psi_{j}(y)\right)_{W_{n}(y)} W_{n}(x), i, j=1,2, \ldots, 2^{k-1} M
$$




\subsection{Operational matrix of the integration}

The integration of the vector $\psi(x)$ defined in 12 can be achieved as

$$
\int_{0}^{x} \psi(t) d t=P \psi(x)
$$

where $P$ is the $2^{k-1} M \times 2^{k-1} M$ operational matrix of integration [10, 11].This matrix is determined as follows

$$
P=\frac{1}{2^{k}}\left[\begin{array}{ccccc}
L & F & F & \cdots & F \\
O & L & F & \ddots & \vdots \\
O & O & L & \ddots & F \\
\vdots & \ddots & \ddots & \ddots & F \\
O & \cdots & O & O & L
\end{array}\right],
$$

where $L, F$ and $O$ are $M \times M$ matrices given by

$$
L=\left[\begin{array}{cccccccc}
1 & \frac{1}{\sqrt{2}} & 0 & & 0 & 0 & \cdots & 0 \\
-\frac{\sqrt{2}}{4} & 0 & \frac{1}{4} & 0 & 0 & \cdots & 0 \\
-\frac{\sqrt{2}}{3} & -\frac{1}{2} & 0 & & \frac{1}{6} & 0 & \cdots & 0 \\
\vdots & \ddots & \ddots & & \ddots & & & \vdots \\
\frac{\sqrt{2}}{2}(-1)^{r}\left(\frac{1}{r-2}-\frac{1}{r}\right) & \ldots & -\frac{1}{2(r-2)} & 0 & \frac{1}{2 r} & \cdots & \\
\vdots & & & \ddots & \ddots & \ddots & & \vdots \\
\frac{\sqrt{2}}{2}(-1)^{M}\left(\frac{1}{M-2}-\frac{1}{M}\right) & 0 & 0 & & 0 & \cdots & -\frac{1}{2(M-2)} & 0
\end{array}\right],
$$




$$
\begin{aligned}
& F=\left[\begin{array}{lrrr}
2 & 0 & \cdots & 0 \\
0 & 0 & \cdots & 0 \\
-\frac{2 \sqrt{2}}{3} & 0 & \cdots & 0 \\
\vdots & \vdots & \ddots & \vdots \\
\frac{\sqrt{2}}{2}\left(\frac{1-(-1)^{r}}{r}-\frac{1-(-1)^{r-2}}{r-2}\right) & 0 & \cdots & 0 \\
\vdots & \vdots & \ddots & \vdots \\
\frac{\sqrt{2}}{2}\left(\frac{1-(-1)^{M}}{M}-\frac{1-(-1)^{M-2}}{M-2}\right) & 0 & \cdots & 0 \\
M & & &
\end{array}\right], \\
& O=\left[\begin{array}{cccc}
0 & 0 & \cdots & 0 \\
0 & 0 & \cdots & 0 \\
\vdots & \vdots & \ddots & \vdots \\
0 & 0 & \cdots & 0
\end{array}\right]
\end{aligned}
$$

The property of the product of two Chebyshev wavelets vector functions will be as follows

$$
\psi(x) \psi^{T}(x) Y \approx \tilde{Y} \psi(x)
$$

where $Y$ is a given vector and $\tilde{Y}$ is a $2^{k-1} M \times 2^{k-1} M$ matrix. This matrix is called the operational matrix of product.

\section{SOLUTION OF SYSTEM OF INTEGRO-DIFFERENTIAL EQUATIONS VIA CHEBYSHEV WAVELETS METHOD}

Consider the system of integral-differential equations (1). Let's consider the following approximation

$$
f_{i}^{\left(m_{i}\right)}(x) \simeq C_{i}^{T} \psi(x), i=1,2, \ldots, n
$$


where $C_{i} i=1,2, \ldots, n$ are $2^{k-1} M \times 1$ matrices given by

$$
\begin{aligned}
C_{i} & =\left[c_{10}^{i}, c_{11}^{i}, \ldots, c_{1 M-1}^{i}, c_{20}^{i}, c_{21}^{i}, \ldots, c_{2 M-1}^{i}, \ldots, c_{2^{k-1} 0}^{i}, \ldots, c_{2^{k-1} M-1}^{i}\right]^{T} \\
& =\left[c_{i, 1}, c_{i, 2}, \ldots, c_{i, M}, c_{i, M+1}, \ldots, c_{i, 2^{k-1} M}\right]^{T}
\end{aligned}
$$

and $\psi(x)$ is defined in (12).

Integrating (22) respect to $x$ from 0 to $x$, we obtain

$$
f_{i}^{(s)}(x) \simeq C_{i}^{T} P^{m_{i}-s} \psi(x)+\sum_{j=0}^{m_{i}-1-s} \alpha_{i, s+j} \frac{x^{j}}{j !}, i=1,2, \ldots, n, s=0,1, \ldots, m_{i}-1
$$

where $P$ is the $2^{k-1} M \times 2^{k-1} M$ operational matrix of integration.

Also consider the following approximations

$$
\begin{aligned}
& g_{i}(x) \simeq G_{i}^{T} \psi(x) \\
& F_{i k}\left(x, f_{1}(x), f_{1}^{\prime}(x), \ldots, f_{1}^{\left(m_{1}\right)}(x), \ldots, f_{n}(x), \ldots, f_{n}^{\left(m_{n}\right)}(x)\right) \simeq X_{i k}^{T} \psi(x), \\
& G_{i j}\left(f_{1}(t), \ldots, f_{1}^{\left(m_{1}\right)}(t), \ldots, f_{n}(t), \ldots, f_{n}^{\left(m_{n}\right)}(t)\right) \simeq Y_{i j}^{T} \psi(t), \\
& k_{i j}(x, t) \simeq \psi^{T}(x) K_{i j} \psi(t) .
\end{aligned}
$$

where $K_{i j}$ are the $2^{k-1} M \times 2^{k-1} M$ matrices, $G_{i}$ are the $2^{k-1} M \times 1$ matrices and $X_{i k}, Y_{i j}$ are column vectors with the entries of the vectors $C_{i}$ for $i=1,2, \ldots, n, j=1,2, \ldots m^{\prime}, k=1,2, \ldots, m$.

Substitution of approximations (22), (24) and (25) into the system (1) will be resulted to: 


$$
\begin{aligned}
G_{i}^{T} \psi(x) & =\sum_{k=1}^{m} X_{i k}^{T} \psi(x)+\sum_{j=1}^{m^{\prime}} \int_{0}^{x} \psi^{T}(x) K_{i j} \psi(t) \psi^{T}(t) Y_{i j} d t \\
& =\sum_{k=1}^{m} X_{i k}^{T} \psi(x)+\sum_{j=1}^{m^{\prime}} \psi^{T}(x) K_{i j}\left(\int_{0}^{x} \psi(t) \psi^{T}(t) Y_{i j} d t\right) \\
& =\sum_{k=1}^{m} X_{i k}^{T} \psi(x)+\sum_{j=1}^{m^{\prime}} \psi^{T}(x) K_{i j} \tilde{Y}_{i j} P \psi(x) \\
i=1,2, \ldots, n &
\end{aligned}
$$

where $\tilde{Y}_{i j}$ are $2^{k-1} M \times 2^{k-1} M$ operational matrices for production and $\mathrm{P}$ is the $2^{k-1} M \times 2^{k-1} M$ operational matrix of integration[10-12]. According to the Galerkin method by multiplying $W_{n}(x) \psi^{T}(x)$ in both sides of the system (26) and then applying $\int_{0}^{x}() d$.$x , linear or non-linear system in terms of the entries of C_{i}$ $, i=1,2, \ldots, n$, will be obtained. The elements of vector functions $C_{i}$ $, i=1,2, \ldots, n$, can be computed by solving these systems. Finally the solution of the system of integral-differential equations 1 will be as follow

$$
f_{i}(x) \simeq C_{i}^{T} P^{m_{i}} \psi(x)+\sum_{j=0}^{m_{i}-1} \alpha_{i, j} \frac{x^{j}}{j !} \quad i=1,2, \ldots, n
$$

\section{NUMERICAL EXAMPLES}

In this section, some examples taken from [17, 18] are considered and will be solved. These examples are solved for $k=1$ and $M=8$.

EXAMPLE 1. In this example the following system of two nonlinear integrodifferential equations with initial conditions $f(0)=1, f^{\prime}(0)=2, g(0)=-1$, and $g^{\prime}(0)=0 \quad$ are $\quad$ studied. The exact solution is $f(x)=x+e^{x} \quad$ and $g(x)=x-e^{x}$. 


$$
\left\{\begin{array}{l}
f^{\prime \prime}(x)+\frac{1}{2} g^{\prime 2}(x)-\frac{1}{2} \int_{0}^{x}\left(f^{2}(t)+g^{2}(t)\right) d t=1-\frac{1}{3} x^{3} \\
g^{\prime \prime}(x)+x f(x)-\frac{1}{4} \int_{0}^{x}\left(f^{2}(t)-g^{2}(t)\right) d t=-1+x^{2}
\end{array}\right.
$$

Let's consider the following approximations

$$
\begin{aligned}
& f^{\prime \prime}(x) \simeq C_{1}^{T} \psi(x), f^{\prime}(x) \simeq C_{1}^{T} P \psi(x)+f^{\prime}(0), f(x) \simeq C_{1}^{T} P^{2} \psi(x)+\left(x f^{\prime}(0)+f(0)\right), \\
& g^{\prime \prime}(x) \simeq C_{2}^{T} \psi(x), g^{\prime}(x) \simeq C_{2}^{T} P \psi(x)+g^{\prime}(0), g(x) \simeq C_{2}^{T} P^{2} \psi(x)+\left(x g^{\prime}(0)+g(0)\right), \\
& g^{\prime 2}(x) \simeq X_{1}^{T} \psi(x), x f(x) \simeq X_{2}^{T} \psi(x), f^{2}(t) \simeq Y_{1}^{T} \psi(t), \\
& g^{2}(t) \simeq Y_{2}^{T} \psi(t), 1-\frac{1}{3} x^{3} \simeq G_{1}^{T} \psi(x),-1+x^{2} \simeq G_{2}^{T} \psi(x) .
\end{aligned}
$$

Substitution into system (28), leads to the following system

$$
\left\{\begin{array}{l}
G_{1}^{T} \psi(x)=C_{1}^{T} \psi(x)+\frac{1}{2} X_{1}^{T} \psi(x)-\frac{1}{2} Y_{1}^{T} P \psi(x)-\frac{1}{2} Y_{2}^{T} P \psi(x) \\
G_{2}^{T} \psi(x)=C_{2}^{T} \psi(x)+X_{2}^{T} \psi(x)-\frac{1}{4} Y_{1}^{T} P \psi(x)+\frac{1}{4} Y_{2}^{T} P \psi(x)
\end{array}\right.
$$

Multiply $W_{n}(x) \psi^{T}(x)$, on both sides of the system 29 applying $\int_{0}^{x}() d$.$x , and$ then solve the system. The elements of vector functions $C_{1}, C_{2}$ can be obtained as follows

$$
\begin{aligned}
\mathrm{C}_{1}= & {[2.197536030,0.7536333811,0.09323771508,0.007729595825,0.0004815902542,} \\
& \left.0.00002402820137,9.995917358 \times 10^{-7}, 5.320378706 \times 10^{-8}\right]^{T},
\end{aligned}
$$

$\mathrm{C}_{2}=[-2.197540267,-0.7536368022,-0.09323821870,-0.007729688661,-0.0004816043689$, $\left.-0.00002403061853,-9.997878544 \times 10^{-7},-3.505832643 \times 10^{-8}\right]^{T}$. 
Therefore, the following solutions will result.

$$
\begin{aligned}
f(x) \simeq & C_{1}^{T} P^{2} \psi(x)+\left(x f^{\prime}(0)+f(0)\right) \\
= & 0.0003297875810 x^{7}+0.001611871153 x^{6}+0.007239035322 x^{5}+0.04299458699 x^{4} \\
& \quad+0.1664423845 x^{3}+0.5001598474 x^{2}+2.000000160 x+0.9999999986, \\
g(x) \simeq & C_{2}^{T} P^{2} \psi(x)+\left(x g^{\prime}(0)+g(0)\right) \\
= & -0.0003300704321 x^{7}-0.001092317748 x^{6}-0.008758021028 x^{5}-0.04138813647 x^{4} \\
- & 0.1669025645 x^{3}-0.5001171685 x^{2}-1.599266077 \times 10^{-7} x-0.9999999986 .
\end{aligned}
$$

Table 1 shows some values of the solutions and absolute errors at some $x$ 's and plots of the exact and approximate solutions are shown in Fig. 1.

Table 1: Numerical results of Example 1.

\begin{tabular}{lllllll}
\hline$x$ & $f$ exact & $f C W M$ & Error $f(x)$ & $g$ exact & $g C W M$ & Error $g(x)$ \\
\hline 0.0 & 1.0000000000 & 0.9999999986 & 0.0000000014 & -1.00000000 & -0.9999999986 & 0.0000000014 \\
0.1 & 1.205170918 & 1.205172429 & 0.000001511 & -1.005170918 & -1.005172316 & 0.000001398 \\
0.2 & 1.421402758 & 1.421409179 & 0.000006421 & -1.021402758 & -1.021409036 & 0.000006278 \\
0.3 & 1.649858808 & 1.649875471 & 0.000016663 & -1.049858808 & -1.049874355 & 0.000015547 \\
0.4 & 1.891824698 & 1.891859882 & 0.000035184 & -1.091824698 & -1.091854807 & 0.000030109 \\
0.5 & 2.148721271 & 2.148786482 & 0.000065211 & -1.148721271 & -1.148772284 & 0.000051013 \\
0.6 & 2.422118800 & 2.422228636 & 0.000109836 & -1.222118800 & -1.222198358 & 0.000079558 \\
0.7 & 2.713752707 & 2.713924633 & 0.000171926 & -1.313752707 & -1.313870048 & 0.000117341 \\
0.8 & 3.025540928 & 3.025795304 & 0.000254376 & -1.425540928 & -1.425707202 & 0.000166274 \\
0.9 & 3.359603111 & 3.359963795 & 0.000360684 & -1.559603111 & -1.559831673 & 0.000228562 \\
1.0 & 3.718281828 & 3.718777672 & 0.000495844 & -1.718281828 & -1.718588437 & 0.000306609
\end{tabular}



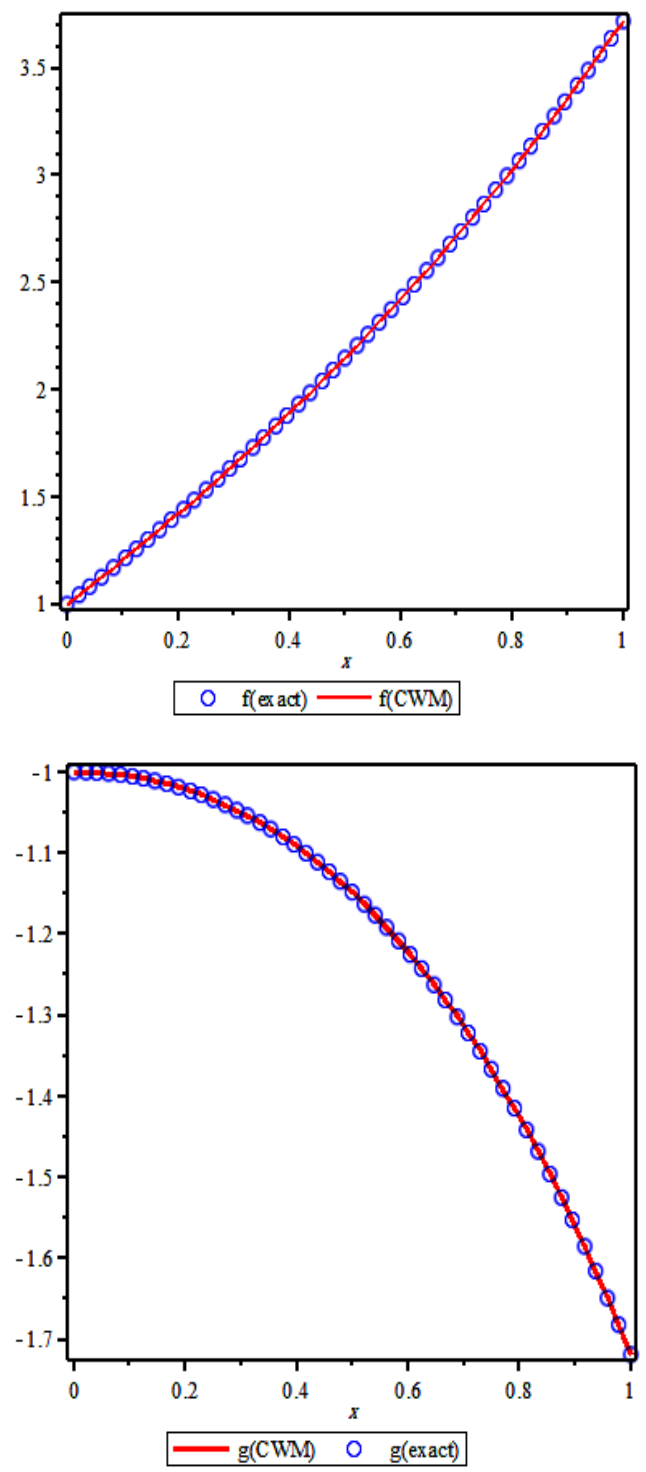

Fig. 1: Comparison of the exact and approximate solution of Example 1. 
EXAMPLE 2. Consider the following nonlinear system of integro-differential equations with the initial condition, $f(0)=f^{\prime \prime}(0)=0, f^{\prime}(0)=1, g(0)=1, g^{\prime}(0)=0$, and $g^{\prime \prime}(0)=-1$.

$$
\left\{\begin{array}{l}
f^{\prime \prime \prime}(x)+f^{\prime}(x)+\int_{0}^{x}\left(f^{\prime \prime 2}(t)+g^{\prime \prime 2}(t)\right) d t=x \\
g^{\prime \prime \prime}(x)-\int_{0}^{x} f^{\prime \prime}(t) g(t) d t=\sin x+\frac{1}{2} \sin ^{2} x
\end{array}\right.
$$

The exact solutions are $f(x)=\sin x$ and $g(x)=\cos x$.

\section{Let's}

$$
\begin{aligned}
& f^{\prime \prime \prime}(x) \simeq C_{1}^{T} \psi(x), f^{\prime \prime}(x) \simeq C_{1}^{T} P \psi(x)+f^{\prime \prime}(0), f^{\prime}(x) \simeq C_{1}^{T} P^{2} \psi(x)+\left(x f^{\prime \prime}(0)+f^{\prime}(0)\right), \\
& f(x) \simeq C_{1}^{T} P^{3} \psi(x)+\left(\frac{1}{2} x^{2} f^{\prime \prime}(0)+x f^{\prime}(0)+f(0)\right), \quad g^{\prime \prime \prime}(x) \simeq C_{2}^{T} \psi(x), g^{\prime \prime}(x) \simeq C_{2}^{T} P \psi(x)+g^{\prime \prime}(0), \\
& g^{\prime}(x) \simeq C_{2}^{T} P^{2} \psi(x)+\left(x g^{\prime \prime}(0)+g^{\prime}(0)\right), g(x) \simeq C_{2}^{T} P^{3} \psi(x)+\left(\frac{1}{2} x^{2} g^{\prime \prime}(0)+x g^{\prime}(0)+g(0)\right), \\
& f^{\prime \prime 2}(t) \simeq Y_{1}^{T} \psi(t), g^{\prime \prime 2}(t) \simeq Y_{2}^{T} \psi(t), f^{\prime \prime}(t) g(t) \simeq Y_{3}^{T} \psi(t), x \simeq G_{1}^{T} \psi(x), \sin x+\frac{1}{2} \sin ^{2}(x) \simeq G_{2}^{T} \psi(x) .
\end{aligned}
$$

The vectors $C_{1}$ and $C_{2}$ are computed by solving the system of nonlinear equations for eight unknowns via the Maple package, as follow

$$
\begin{aligned}
\mathrm{C}_{1}= & {[-1.033178901,0.2054258148,0.04759333273,-0.002203298247,-0.0002500559039,} \\
& \left.0.000005544755581,7.071954337 \times 10^{-7},-2.113621989 \times 10^{-7}\right]^{T}, \\
C_{2}= & {[0.5635357589,0.3754607061,-0.02616464781,-0.004512844558,0.0001094937002,} \\
& \left.-0.00009332958970,-0.000005016576345,5.596357236 \times 10^{-7}\right]^{T} .
\end{aligned}
$$

Therefore, we have the following approximate solutions 


$$
\begin{aligned}
f(x) \simeq & C_{1}^{T} P^{3} \psi(x)+\left(\frac{1}{2} x^{2} f^{\prime \prime}(0)+x f^{\prime}(0)+f(0)\right) \\
= & -0.0001728157537 x^{7}-0.00006176762956 x^{6}+0.008404014732 x^{5}-0.00008238957098 x^{4} \\
& -.1667033953 x^{3}-7.511600124 \times 10^{-7} x^{2}+1.000000037 x-3.020407080 \times 10^{-10}, \\
g(x) \simeq & C_{2}^{T} P^{3} \psi(x)+\left(\frac{1}{2} x^{2} g^{\prime \prime}(0)+x g^{\prime}(0)+g(0)\right), \\
= & 0.00008122166633 x^{7}-0.001581825515 x^{6}+0.0003131253525 x^{5}+0.04134048203 x^{4} \\
& +0.0001944003421 x^{3}-0.4999871714 x^{2}-6.242933536 \times 10^{-7} x+1.000000005 .
\end{aligned}
$$

Table 2 shows some values of the solutions and absolute errors at some $x$ 's and plots of the exact and approximate solutions are shown in Fig. 2. In this example we use the Taylor expansion of $\left(\sin x+\frac{1}{2} \sin ^{2} x\right)$ about the point $x=\frac{1}{2}$.

Table 2: Numerical results of Example 2.

\begin{tabular}{lllllll}
\hline$x$ & $f$ exact & $f C W M$ & Error $f(x)$ & $g$ exact & $g C W M$ & Error $g(x)$ \\
\hline 0.0 & 0.000000 & $-3.0204 \times 10^{-10}$ & $-3.0204 \times 10^{-10}$ & 1.00000000 & 1.000000005 & 0.000000005 \\
0.1 & 0.09983341665 & 0.09983336822 & 0.00000004843 & 0.9950041653 & 0.9950044009 & 0.0000002356 \\
0.2 & 0.1986693308 & 0.1986689012 & 0.0000004296 & 0.9800665778 & 0.9800680933 & 0.0000015155 \\
0.3 & 0.2955202067 & 0.2955186231 & 0.0000015836 & 0.9553364891 & 0.9553407045 & 0.0000042154 \\
0.4 & 0.3894183423 & 0.3894142888 & 0.0000040535 & 0.9210609940 & 0.9210694261 & 0.0000084321 \\
0.5 & 0.4794255386 & 0.4794170669 & 0.0000084717 & 0.8775825619 & 0.8775966840 & 0.0000141221 \\
0.6 & 0.5646424734 & 0.5646269170 & 0.0000155564 & 0.8253356149 & 0.8253567863 & 0.0000211714 \\
0.7 & 0.6442176872 & 0.6441915749 & 0.0000261123 & 0.7648421873 & 0.7648715988 & 0.0000294115 \\
0.8 & 0.7173560909 & 0.7173150568 & 0.0000410341 & 0.6967067093 & 0.6967452826 & 0.0000385733 \\
0.9 & 0.7833269096 & 0.7832655972 & 0.0000613124 & 0.6216099683 & 0.6216581409 & 0.0000481726 \\
1.0 & 0.8414709848 & 0.8413829320 & 0.0000880528 & 0.5403023059 & 0.5403596132 & 0.0000573073
\end{tabular}



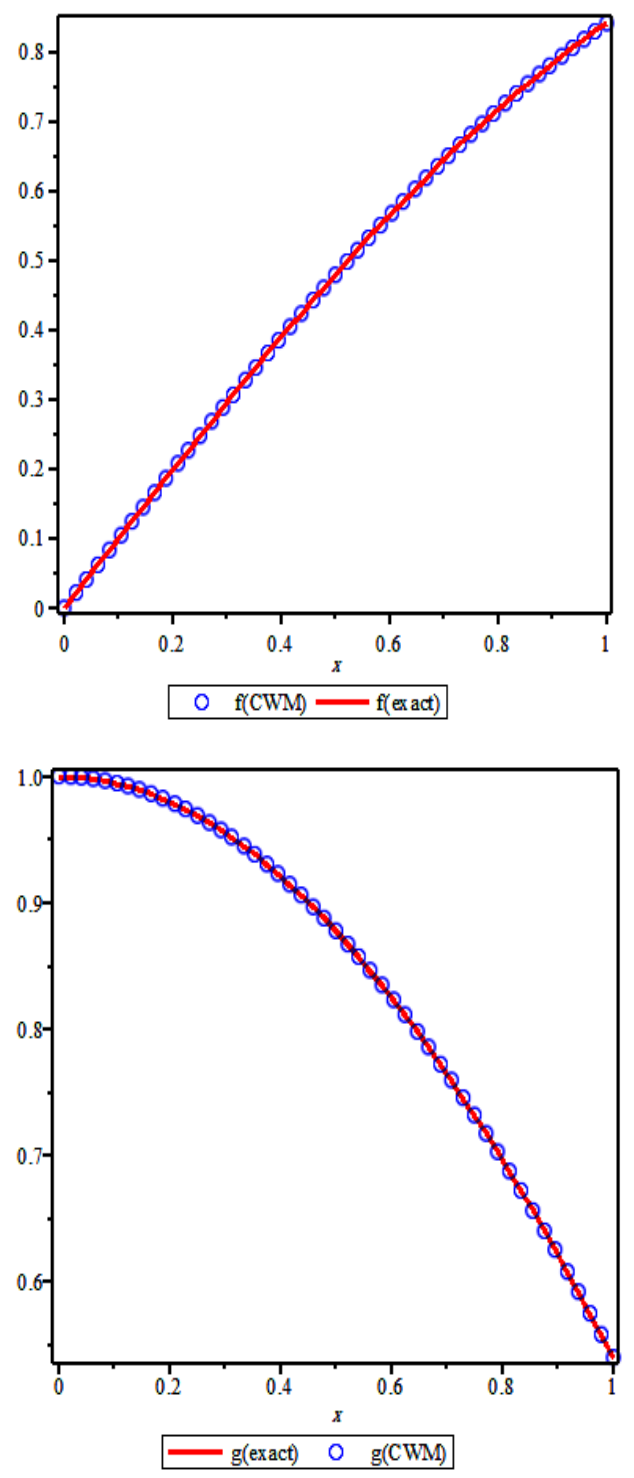

Fig. 2: Comparison of the exact and approximate solution of Example 2. 
EXAMPLE 3. Consider the following nonlinear of integral-differential equations, with the initial conditions

$$
\left\{\begin{array}{l}
f^{\prime}(x)+\frac{1}{2} g^{\prime 2}(x)-\int_{0}^{x}((x-t) f(t)+f(t) g(t)) d t=\cosh x+x-\sinh x \\
g^{\prime}(x)-\int_{0}^{x}\left((x-t) f(t)-g^{2}(t)+f^{2}(t)\right) d t=2 x .
\end{array}\right.
$$

for which the exact solution is $f(x)=\sinh x$ and $g(x)=\cosh x$.

Let's consider the following approximations

$$
\begin{aligned}
& f^{\prime}(x) \simeq C_{1}^{T} \psi(x), f(x) \simeq C_{1}^{T} P \psi(x)+f(0), g^{\prime}(x) \simeq C_{2}^{T} \psi(x), g(x) \simeq C_{2}^{T} P \psi(x)+g(0), \\
& g^{\prime 2}(x) \simeq X_{1}^{T} \psi(x), f(t) g(t) \simeq Y_{1}^{T} \psi(t), f^{2}(t) \simeq Y_{2}^{T} \psi(t), g^{2}(t) \simeq Y_{3}^{T} \psi(t), \\
& \cosh x+x-\sinh x \simeq G_{1}^{T} \psi(x), 2 x \simeq G_{2}^{T} \psi(x),(x-t) \simeq \psi^{T}(x) K \psi(t) .
\end{aligned}
$$

by applying the Chebyshev wavelets method and solving the resulted nonlinear system, the following results would be achieved.

$$
\begin{aligned}
C_{1}= & {[1.504165432,0.2389528422,0.06386953494,0.002378988860,0.0003125907180,} \\
& \left.0.000005695406017,6.628263669 \times 10^{-7}, 9.271731628 \times 10^{-8}\right]^{T}, \\
C_{2}= & {[0.6943499315,0.5155176669,0.02974272548,0.005344707710,0.0001561907394,} \\
& \left.0.00001618917558,9.368339090 \times 10^{-8},-1.237827103 \times 10^{-8}\right]^{T} .
\end{aligned}
$$

Therefore, we have the following approximate solutions 


$$
\begin{aligned}
f(x) \simeq & C_{1}^{T} P \psi(x)+f(0) \\
& =0.0002188198816 x^{7}-0.0002263959022 x^{6}+0.008445156702 x^{5}+2.522934920 \times 10^{-7} x^{4} \\
& +0.1674136595 x^{3}-6.443851028 \times 10^{-7} x^{2}+1.000252973 x-3.269384483 \times 10^{-9} . \\
g(x) \simeq & C_{2}^{T} P \psi(x)+g(0) \\
= & 0.00003092782758 x^{7}+0.001451773568 x^{6}-0.00002212408164 x^{5}+0.04199155669 x^{4} \\
- & 0.000006081823674 x^{3}+0.4993663134 x^{2}-5.238903274 \times 10^{-8} x+1.000000000 .
\end{aligned}
$$

Some values of exact, approximate solutions and absolute errors are presented in Table 3 and plots of exact and approximate solutions are shown in Fig. 3. In this example we use the Taylor expansion of $(\cosh x+x-\sinh x)$ about the point $x=\frac{1}{2}$

Table 3: Numerical results of Example 3.

\begin{tabular}{ccccccc}
\hline$x$ & $f$ exact & $f C W M$ & Error $f(x)$ & $g$ exact & $g$ CWM & Error $g(x)$ \\
\hline 0.0 & 0.00000 & $-3.2694 \times 10^{-9}$ & $3.2694 \times 10^{-9}$ & 1.00000000 & 1.000000000 & 0.00000000 \\
0.1 & 0.1001667500 & 0.1001927855 & 0.0000260355 & 1.005004168 & 1.004997852 & 0.000006316 \\
0.2 & 0.2013360025 & 0.2013925660 & 0.0000565635 & 1.020066756 & 1.020041866 & 0.000024890 \\
0.3 & 0.3045202934 & 0.3046164060 & 0.0000961126 & 1.045338514 & 1.045283931 & 0.000054583 \\
0.4 & 0.4107523258 & 0.4109014731 & 0.0001491473 & 1.081072372 & 1.080978954 & 0.000093418 \\
0.5 & 0.5210953055 & 0.5213151285 & 0.0002198230 & 1.127625965 & 1.127487498 & 0.000138467 \\
0.6 & 0.6366535821 & 0.6369651899 & 0.0003116078 & 1.185465218 & 1.185279513 & 0.000185705 \\
0.7 & 0.7585837018 & 0.7590104708 & 0.0004267690 & 1.255169006 & 1.254939172 & 0.000229834 \\
0.8 & 0.8881059822 & 0.8886717102 & 0.0005657280 & 1.337434946 & 1.337170837 & 0.000264109 \\
0.9 & 1.026516726 & 1.027242999 & 0.000726273 & 1.433086385 & 1.432806154 & 0.000280231 \\
1.0 & 1.175201194 & 1.176103818 & 0.000902624 & 1.543080635 & 1.542812313 & 0.000268322
\end{tabular}



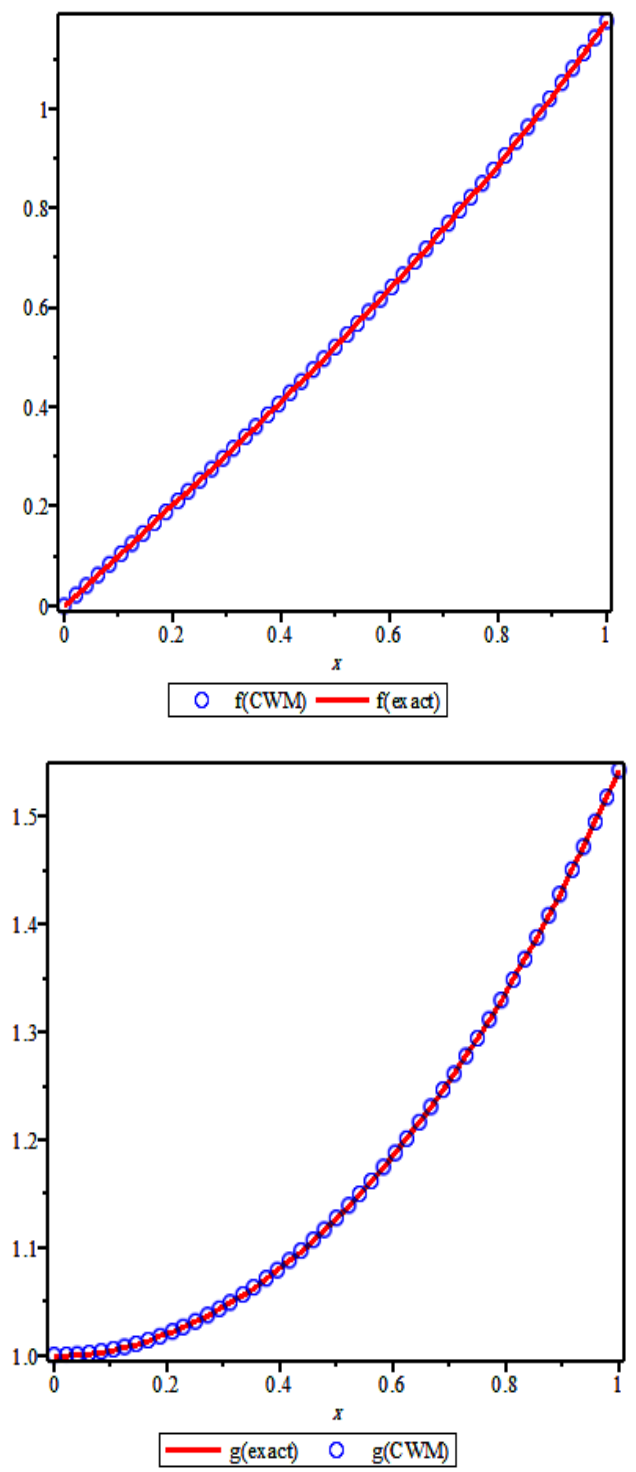

Fig. 3: Comparison of the exact and approximate solution of Example 3. 


\section{CONCLUSION}

In this paper, the Chebyshev wavelets method for the solution of system of nonlinear integro-differential equations is successfully expanded. Illustrative examples are included to demonstrate that the method is a very useful technique for finding approximate solutions of these systems. This technique is a powerful tool for solving various systems of integral and integro-differential equations. The package Maple 16 is used for computation.

\section{REFERENCES}

[1] M. Dehghan, F. Shakeri, Solution of an integro-differential equation arising in oscillating magnetic fields using He's homotopy perturbation method, Prog. Electromagn. Res., 78 (2008) 361-376.

[2] A. M. Siddiqui, R. Mahmood, G.K. Ghori, Homotopy perturbation method for thin film flow of a fourth grade fluid down a vertical cylinder, Phys. Lett. A, 352 (2006) 404-410.

[3] K. M. Tamizhmani, J. Satsuma, B. Grammaticos, V. Ramani, Nonlinear integro differential equations as a discreet systems, Inverse Probl., 15 (1999) 787-791.

[4] L. Xu, J. H. He, Y. Liu, Electrospun nanoporous spheres with Chinese drug, International Journal of Nonlinear Sciences and Numerical Simulation, 8 (2007) 199-202.

[5] H. Wang, H.M. Fu, H.F. Zhang, A practical thermodynamic method to calculate the best glassforming composition for bulk metallic glasses, International Journal of Nonlinear Sciences and Numerical Simulation, 8 (2007) 171-178.

[6] F. Z. Sun, M. Gao, S. H. Lei, The fractal dimension of the fractal model of dropwise condensation and its experimental study, International Journal of Nonlinear Sciences and Numerical Simulation, 8 (2007) 211-222.

[7] T. L. Bo, L. Xie, X.LJ. Zheng, Numerical approach to wind ripple in desert, International Journal of Nonlinear Sciences and Numerical Simulation, 8 (2007) 223-228.

[8] H. Brunner, Collocation Method for Volterra Integral and Related Functional Equations, Cambridge Monograph on Applied and Computational Mathematics, Cambridge University Press, Cambridge, MA, 2004.

[9] Y. LI, Solving a nonlinear fractional differential equation using Chebyshev wavelets, Communications in Nonlinear Science and Numerical Simulation 15 (2010) 2284-2292.

[10] E. Babolian, F. Fattahzadeh, Numerical computation method in solving integral equations by using Chebyshev wavelet operational matrix of integration, Applied Mathematics and Computations 188 (2007) 1016-1022.

[11] E. Babolian, F. Fattahzadeh, Numerical solution of differential equations by using Chebyshev wavelet operational matrix of integration, Applied Mathematics and Computations 188 (2007) 417-426.

[12] Y. LI, Solving a nonlinear fractional differential equation using Chebyshev wavelets, Communications in Nonlinear Science and Numerical Simulation 15 (2010) 2284-2292.

[13] Ole Christensen, Khadjia L. Christensen, Approximation Theory: from Taylor Polynomial to Wavelets, Birkhauser, Boston, 2004.

[14] I. Daubeches, Ten lectures on wavelets, CBMS-NSF, 1992.

[15] D. Gottlieb, S.A. Orszag, Numerical Analysis of Spectral Methods, SIAM, Philadelphia, PA, 1997.

[16] J.C. Goswami, Chan, Fundamentals of Wavelets. Theory, Algorithms, and Applications, John Wiley and Sons, New York, 1999. 
[17] H. Aminikhah, M. Salahi, A new homotopy perturbation method for system of nonlinear integrodifferential equations, International Journal of Computer Mathematics, 87 (2010) 1186-1194.

[18] J. Biazar, H. Aminikhah, A new technique for solving nonlinear integral_differential equations, Computers and Mathematics with Applications 58 (2009) 2084-2090.

H. Aminikhah

Department of Applied Mathematics, School of Mathematical Sciences, University of Guilan,

P.O. Box 1914, P.C. 41938, Rasht, Iran

Corresponding author: Tel/fax: +98 1313233509

E-mail address: hossein.aminikhah@gmail.com, aminikhah@guilan.ac.ir

\section{S. Hosseini}

Department of Applied Mathematics, School of Mathematical Sciences, University of Guilan, P.O. Box 1914, P.C. 41938, Rasht, Iran

Corresponding author: Tel/fax: +98 1313233509

E-mail address: hoseyni_sahar11@yahoo.com 